\title{
Un análisis de hábitos de estudio y rendimiento académico en el curso de matemática de los estudiantes del $6^{\circ}$ grado de primaria
}

\author{
Study habits and academic performance, in the area of mathematics of \\ students of the 6th grade of primary school \\ Russ Williams Acuña Casaico ${ }^{1 \mathrm{a}}$ \\ Universidad Peruana Unión, Lima-Perú ${ }^{1}$
}

Recibido: 04 de julio de 2020

Aceptado: 23 de diciembre de 2020

\section{Resumen}

El presente estudio aborda los hábitos de estudio y el rendimiento académico en el área de matemática, cuyos estudiantes participantes procedieron de instituciones educativas adventistas, adscritas en el departamento de San Martín, con el propósito de determinar si existe relación significativa entre las variables, cuya investigación presenta el enfoque cuantitativo, y su diseño fue no experimental, de tipo correlacional. La muestra estuvo constituida por 71 estudiantes del sexto grado de primaria. Se trabajó el cuestionario de tipo Lickert, para la variable hábitos de estudio. Los resultados, para la segunda variable, se obtuvieron a partir de las actas de evaluación, y se trabajó el coeficiente de correlación Rho Spearman, con el propósito de realizar la prueba de las hipótesis. Se concluye que la relación entre hábitos de estudio y rendimiento académico no es significativa.

Palabras clave: hábitos de estudio, rendimiento académico, ambiente de estudio

\begin{abstract}
This research addresses study habits and academic performance, in the area of mathematics, whose participating students were from Adventist educational institutions, assigned in the department of San Martín, with the purpose of determining if there is a significant relationship of the variables, whose Research presents the quantitative approach, whose design was non-experimental, correlational. The sample consisted of 71 students in the sixth grade of primary school. The Lickert type questionnaire was worked for the study habits variable. The results, for the second variable, the evaluation records were used, the correlation coefficient Rho Spearman was worked, in order to test the hypotheses. It is concluded that the relationship is not significant.
\end{abstract}

Keywords: study habits, academic performance, study environment.

${ }^{\mathrm{a} C}$ Correspondencia al autor:

E-mail: willieducprim@gmail.com 


\section{Introducción}

La insatisfacción de los padres de familia con el bajo rendimiento académico de los estudiantes en las instituciones educativas del departamento de San Martín (Perú), se constituye en el problema de investigación y nace con el objetivo de determinar la relación existente entre las variables mencionadas. Los hábitos de estudio son fundamentales para el éxito de los estudiantes en la escuela; la organización personal del estudiante (cómo estudiar, dónde estudiar, cuándo estudiar, etc.) y la ejecución de las tareas en sus respectivos domicilios, facilitan la buena calidad del aprendizaje. El rendimiento académico, en el área de matemática, es uno de los aspectos más controvertidos del proceso educativo. Así surge el interés en investigar si los hábitos de estudio se relacionan o no con el rendimiento académico en el área de matemática.

\section{Importancia de los hábitos de estudio}

Cierto número de autores analizan la importancia de los hábitos de lectura desde diferentes áreas de abordaje, como Pedreros Herrera (2014), quienes argumentan que los hábitos de lectura posibilitan los buenos resultados académicos y la retención de la información. También, los hábitos de estudio mejoran la capacidad de retención de información en las técnicas de estudio (Vera, 1996; Torres, 2008). Según Rosales Fernandez (2016), los hábitos de estudio benefician la capacidad intelectual, que se reflejan en un buen rendimiento académico (Maddox, 1970; Torres, 2008; Grajales, 2002, en Rosales Fernandez, 2016).

Asimismo, los hábitos de estudio permiten un aprendizaje significativo y el desarrollo adecuado de habilidades; por ejemplo, "la planificación, organización, distribución del tiempo, resumir, sintetizar y esquematizar los contenidos e información vista en las áreas de conocimiento, lo que contribuye a la mejora de los resultados académico y de atención" (Torres Bautista, 2017, p. 22). Del mismo modo, los habitos de estudio persiguen la formación multifuncional de los estudiantes, la cual debe ser integral, y realizar cambios en las formas de pensar, observar, concentrarse y organizar conductas que le permitan al estudiante realizar la labor intelectual necesaria para resolver un problema, reflexionar sobre una pregunta y seleccionar estrategias para ejecutar una tarea (Méndez Rodríguez, 2017). 


\section{Rendimiento académico}

Para Barrios-Gaxiola y Frías-Armenta (2016), el rendimiento académico consiste en un proceso psicosocial, determinado por aspectos contextuales, sociales, ambientales escolares, y familiares. Por su parte, Sánchez-Balcázar et al. (2016) consideran que el rendimiento académico se traduce en fracaso o éxito durante el proceso de estudio, cuyo triunfo se determina mediante las notas y calificaciones.

Desde otro aspecto, los hábitos de estudio constituyen un factor elemental para mejorar el rendimiento académico (Gonzalez vda de Antillón, 1985; Sánchez-Balcázar, Flores-Nicolalde, y Flores-Nicolalde, 2016). En este caso, la inteligencia emocional y las inteligencias múltiples juegan un rol excesivo sobre el desempeño académico (Quintanilla Padilla y Tacuri Carrazco, 2011; Sánchez-Balcázar et al., 2016).

Para Vergel-Ortega, Martínez-Lozano, y Zafra-Tristancho (2016), los componentes asociados al rendimiento académico que logran una repercusión considerable, son: la motivación familiar, la conciencia, el tipo de inteligencia, y el tipo y estilo de aprendizaje" (p. 213). Asimismo, juegan un papel importante la organización familiar, las condiciones económicas de la familia, la cultura familiar, entre otros, emiten efectos sobre el logro de los estudiantes. De igual manera, la educación de los padres, sus valores, y sus conocimientos inciden en el desempeño escolar de los menores (Chaparro, González, y Caso, 2016).

Según Vargas-Hernández y Montero-Rojas (2016), no puede quedar al margen el rol de los cursos, la figura de la institución educativa, los exámenes estandarizados, las evaluaciones y el papel de los docentes. Por ello, se incide en que son los padres de familia quienes tienen el papel primordial de construir un ambiente favorable, adecuado, ameno, mucho más propicio y eficaz, para el aprendizaje de sus hijos (Sánchez Balcázar et al., 2016).

\section{Metodología}

El diseño de la investigación es no experimental, de tipo correlacional, porque tiene como propósito medir el grado de relación que existe entre dos o más variables; además estos estudios miden las dos o más variables que se pretenden ver si están o no relacionados en los mismos sujetos (Hernández, Fernández y Baptista, 2014). El objetivo de este estudio es comprobar la posible relación significativa entre las variables de "hábitos de estudio" y "rendimiento académico" de los estudiantes del $6^{\text {to }}$ grado de nivel 
primario, de las Instituciones Educativas Adventistas del Departamento de San Martín, 2018.

Para el desarrollo de la investigación se llevó a cabo las siguientes acciones: a). Se identificó el problema a investigar en las instituciones mencionadas. b) Se identificó la problemática general y análisis del instrumento para la evaluación respectiva. c). Se recopiló los datos del post-test sobre la variable dependiente.

Según los análisis de la investigación sobre el post test, se observó que los niños presentaron problemas en sus calificaciones (bajo rendimiento en el área de matemática). Pues se utilizó el SPSS para los análisis estadísticos, se creó tablas y figuras, para ver estos datos se ingresó datos recopilados y pasaron a ser procesados, llegando a la conclusión con ayuda de un estadista. Se establecieron conclusiones a partir de los objetivos e hipótesis planteadas para el estudio de la investigación y finalmente se adjuntará recomendaciones que serán bases para investigaciones posteriores.

\section{Resultados}

Prueba de correlación del ambiente de estudio y el rendimiento académico

Tabla 1.

Prueba de Correlación Rho de Spearman entre el ambiente de estudio y el rendimiento académico

\begin{tabular}{lcccc}
\hline \multicolumn{4}{c}{ Rendimiento académico } \\
\hline & $\begin{array}{c}\text { Rho de } \\
\text { Spearman }\end{array}$ & $\begin{array}{c}\text { Sig. } \\
\text { (bilateral) }\end{array}$ & $\mathrm{N}$ \\
\hline $\begin{array}{c}\text { Ambiente } \\
\text { estudio }\end{array}$ & de & .119 & .323 & 71 \\
\hline
\end{tabular}

En la Tabla 1 se muestra la relación entre el ambiente de estudio y el rendimiento académico. A través de la aplicación del estadístico de correlación de Rho Spearman se arrojó un resultado de 0,119 y un p valor igual a 0,323 ( $\mathrm{p}$ valor > 0.05); se acepta la hipótesis nula; dicho de otra forma, no existe relación significativa entre el ambiente de estudio y el rendimiento académico en estudiantes del $6^{\circ}$ grado de primaria de las Instituciones Educativas Privadas Adventistas del departamento de San Martín. Por otro lado, se puede ver que la relación existente es directa; es decir, a mejor ambiente de estudio, mayor será el rendimiento académico. 
Tabla 2.

Prueba de Correlación Rho de Spearman entre la organización de actividades y tiempo y el rendimiento académico

\begin{tabular}{lccc}
\hline & \multicolumn{3}{c}{ Rendimiento académico } \\
\hline & $\begin{array}{c}\text { Rho de } \\
\text { Spearman }\end{array}$ & $\begin{array}{c}\text { Sig. } \\
\text { (bilateral) }\end{array}$ & $\mathrm{N}$ \\
\hline $\begin{array}{c}\text { Organización de actividades y } \\
\text { tiempo }\end{array}$ & .118 & .326 & 71 \\
\hline
\end{tabular}

En la Tabla 2 se muestra la relación entre la organización de actividades y tiempo y el rendimiento académico. A través de la aplicación del estadístico de correlación de Rho Spearman se arrojó un resultado de 0,118 y un p valor igual a 0,326 ( $\mathrm{p}$ valor $>0.05$ ); es así que, se acepta la hipótesis nula; dicho de otra forma, no existe relación significativa entre la organización de actividades y tiempo y el rendimiento académico en estudiantes del $6^{\circ}$ grado de primaria de las Instituciones Educativas Privadas Adventistas del departamento de San Martín. Por otro lado, se puede ver que la relación existente es directa; es decir, a mejor organización de actividades y tiempo, mayor será el rendimiento académico.

Prueba de correlación entre las técnicas de estudio y el rendimiento académico

Tabla 3.

Prueba de Correlación Rho de Spearman entre las técnicas de estudio y el rendimiento académico

\begin{tabular}{|c|c|c|c|}
\hline & \multicolumn{3}{|c|}{ Rendimiento académico } \\
\hline & $\begin{array}{l}\text { Rho de } \\
\text { Spearman }\end{array}$ & $\begin{array}{c}\text { Sig. } \\
\text { (bilateral) }\end{array}$ & $\mathrm{N}$ \\
\hline $\begin{array}{l}\text { Técnicas de } \\
\text { estudio }\end{array}$ & .004 & .975 & 71 \\
\hline
\end{tabular}

En la Tabla 3 se muestra la relación entre las técnicas de estudio y el rendimiento académico. A través de la aplicación del estadístico de correlación de Rho Spearman se arrojó un resultado de 0,004 y un p valor igual a 0,975 (p valor > 0.05); es así que, se acepta la hipótesis nula; dicho de otra forma, no existe relación significativa entre las técnicas de estudio y el rendimiento académico en estudiantes del $6^{\circ}$ grado de primaria 
de las Instituciones Educativas Privadas Adventistas del departamento de San Martín. Por otro lado, se puede ver que la relación existente es directa; es decir, a mejores técnicas de estudio, mayor será el rendimiento académico.

\section{Prueba de correlación entre la preparación para los exámenes y el rendimiento académico}

Tabla 4.

Prueba de Correlación Rho de Spearman entre la preparación para los exámenes y el rendimiento académico

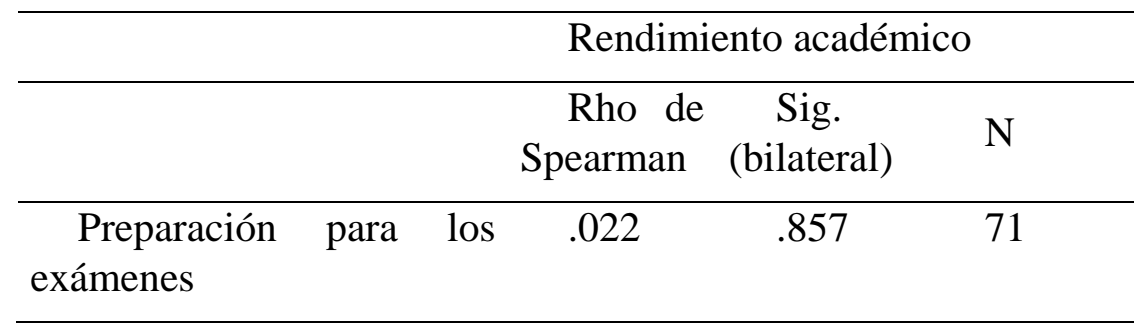

En la Tabla 4 se muestra la relación entre la preparación para los exámenes y el rendimiento académico. A través de la aplicación del estadístico de correlación de Rho Spearman se arrojó un resultado de 0,022 y un p valor igual a 0,857 (p valor > 0.05); es así que, se acepta la hipótesis nula; dicho de otra forma, no existe relación significativa entre la preparación para los exámenes y el rendimiento académico en estudiantes del $6^{\circ}$ grado de primaria de las Instituciones Educativas Privadas Adventistas del departamento de San Martín. Por otro lado, se puede ver que la relación existente es directa; es decir, a mejor preparación para los exámenes, mayor será el rendimiento académico.

Prueba de correlación entre la motivación para el estudio y el rendimiento académico

Tabla 5

Prueba de Correlación Rho de Spearman entre la motivación para el estudio y el rendimiento académico

\begin{tabular}{lccc}
\hline & \multicolumn{3}{c}{ Rendimiento académico } \\
\hline & $\begin{array}{c}\text { Rho de } \\
\text { Spearman }\end{array}$ & $\begin{array}{c}\text { Sig. } \\
\text { (bilateral) }\end{array}$ & $\mathrm{N}$ \\
\hline $\begin{array}{c}\text { Motivación para el } \\
\text { estudio }\end{array}$ & .019 & .877 & 71 \\
\hline
\end{tabular}


En la Tabla 5 se muestra la relación entre la motivación para el estudio y el rendimiento académico. A través de la aplicación del estadístico de correlación de Rho Spearman se arrojó un resultado de 0,019 y un p valor igual a 0,887 ( $\mathrm{p}$ valor > 0.05); es así que, se acepta la hipótesis nula; dicho de otra forma, no existe relación significativa entre la motivación para el estudio y el rendimiento académico en estudiantes del $6^{\circ}$ grado de primaria de las Instituciones Educativas Privadas Adventistas del departamento de San Martín. Por otro lado, se puede ver que la relación existente es directa; es decir, a mejor motivación para el estudio, mayor será el rendimiento académico.

\section{Prueba de correlación entre los hábitos de estudio y el rendimiento académico}

Tabla 6

Prueba de Correlación Rho de Spearman entre los hábitos de estudio y el rendimiento académico

\begin{tabular}{|c|c|c|c|}
\hline & \multicolumn{3}{|c|}{ Rendimiento académico } \\
\hline & $\begin{array}{l}\text { Rho de } \\
\text { Spearman }\end{array}$ & $\begin{array}{c}\text { Sig. } \\
\text { (bilateral) }\end{array}$ & $\mathrm{N}$ \\
\hline $\begin{array}{l}\text { Hábitos de } \\
\text { estudio }\end{array}$ & .094 & .434 & 71 \\
\hline
\end{tabular}

En la Tabla 6 se muestra la relación entre los hábitos de estudio y el rendimiento académico. A través de la aplicación del estadístico de correlación de Rho Spearman se arrojó un resultado de 0,094 y un p valor igual a 0,434 (p valor > 0.05); se acepta la hipótesis nula; dicho de otra forma, no existe relación significativa entre los hábitos de estudio y el rendimiento académico en estudiantes del $6^{\circ}$ grado de primaria de las Instituciones Educativas Privadas Adventistas del departamento de San Martín. Por otro lado, se puede ver que la relación existente es directa; es decir, a mejores hábitos de estudio, mayor será el rendimiento académico.

Se concluye que existe correlación positiva media entre los hábitos de estudio y el nivel de rendimiento académico, en el área de matemática de los estudiantes del 6to grado de primaria de las Instituciones Educativas Adventistas del Departamento de San Martín, 2018.

\section{Conclusión}

El presente trabajo de campo se orientó a examinar el logro de los objetivos planteados en la investigación, cuyo propósito principal fue establecer la posible relación 
entre los hábitos de estudio y el respectivo rendimiento académico en el campo de matemática, en los estudiantes del 6to grado de educación primaria de las Instituciones Educativas Adventistas del Departamento de San Martín, 2018. El instrumento utilizado para recolectar los datos en relación a la variable "hábitos de estudio" muestra un notable patrón de confiabilidad, lo que podemos dirige a afirmar que resulta adecuada su aplicación en la muestra y/o población de estudio.

Asimismo, se ha pretendido asimilar la relación entre el ambiente de estudio y el rendimiento académico, encontrado el estadístico de correlación de Rho Spearman, cuyo resultado es 0,119 y un $\mathrm{p}$ valor igual a 0,323 ( $\mathrm{p}$ valor $>0.05$ ); con ello, se acepta la hipótesis nula; y se concluye que no existe alguna relación significativa entre dichas variables. Por otro lado, se puede observar que la relación existente es de tipo directo; es decir, a mejor ambiente de estudio, mayor será el desempeño académico.

En fin, culminando el análisis de la relación entre los hábitos de estudio y el rendimiento académico, se puede concluir que dicha relación existente es directa; lo que equivale a sostener que, a mejores hábitos de estudio, mayor será el rendimiento académico. No obstante, a pesar de que la relación no es significativa—pues el estadístico de correlación de Rho Spearman dejó un resultado de 0,094 y un p valor igual a 0,434 (p valor > 0.05)— _e resuelve que hay una razón por la cual se acepta la hipótesis nula.

\section{Referencias}

Barreto-Trujillo, F. J., \& Álvarez Bermúdez, J. (2017). Clima escolar y rendimiento académico en estudiantes de preparatoria. Daena: International Journal of Good Conscience, 12(2), 31-44. Recuperado de: http://www.spentamexico.org/v12-n2/A2.12(2)31-44.pdf

Barrios Gaxiola, M. I., \& Frías Armenta, M. (2016). Factores que Influyen en el Desarrollo y Rendimiento Escolar de los Jóvenes de Bachillerato. Revista Colombiana de Psicología, 25(1), 63-82. https://doi.org/10.15446/rcp.v25n1.46921

Capdevila-Seder, A., \& Bellmunt-Villalonga, H. (2016). Importancia de los hábitos de estudio en el rendimiento académico del adolescente: diferencias por género. Educatio Siglo XXI, 34(1), 157-172. Recuperado de: https://www.researchgate.net/publication/313864438_Importancia_de_los_h abitos_de_estudio_en_el_rendimiento_academico_del_adolescente_diferenci as_por_genero

Chaparro, A., González, C., and Caso, J. (2016). Familia y rendimiento académico: configuración de perfiles estudiantiles en secundaria. Revista Electronica de Investigacion Educativa, 18(1), 53-68. Recuperado de http://redie.uabc.mx/redie/article/view/774

Chilca Alva, M. L. (2017). Autoestima , hábitos de estudio y rendimiento académico 
en estudiantes universitarios. Propósitos Y Representaciones, 5(1), 71-127. Recuperado de: https://dialnet.unirioja.es/servlet/articulo?codigo=5904759

Flores Figueredo, N. (2018). Hábitos de estudio en los estudiantes del V ciclo de Educación Primaria de la Institución Educativa $N^{\circ} 70663$ "Carlos Dante Nava Silva”, Juliaca, 2018 (Tesis para el título profesional). Universidad Peruana Unión, Juliaca, Perú. Recuperado de: https://repositorio.upeu.edu.pe/bitstream/handle/UPEU/1643/Nelia_Tesis_Li cenciatura_2018.pdf? sequence $=1 \&$ isAllowed $=\mathrm{y}$

Gonzalez-Antillón, A. (1985). Los hábitos de estudio y su relación con el rendimiento académico. Universidad Francisco Marroquín, Facultad de Humanidades, Departamento de Piscología.

Hereira Ortiz, A. M. (2017). Hábitos de estudio y rendimiento académico en estudiantes del grado undécimo de dos colegios adventistas del norte de Colombia. Memorias, 14(1), 40-53. Recuperado de: http://dspace.biblioteca.um.edu.mx/xmlui/bitstream/handle/20.500.11972/73 2/Tesis\%20Alix\%20Mar\%c3\%ada\%20Hereira\%20Ortiz.pdf?sequence=1\&is Allowed=y

López Pérez, L. V. (2018). Los hábitos de estudio de los estudiantes del IV ciclo de la Institución Educativa Agropecuario $N^{\circ} 15$ - 20347, Santa María, 2018. (Tesis de título profesional). Universidad Nacional José Faustino Sánchez Carrión, Facultad de Educación. Huacho, Perú. Recuperado de: http://repositorio.unjfsc.edu.pe/bitstream/handle/UNJFSC/2743/LOPEZ\%20 PEREZ\%20LESLIE.pdf?sequence $=1 \&$ isAllowed $=\mathrm{y}$

Méndez Rodríguez, E. (2017). Los hábitos de estudio y su relación con el rendimiento académico de los estudiantes de la Institución Educativa $N^{\circ} 821150$ Campo Alegre, distrito Eduardo Villanueva, provincia de San Marcos, 2014. (Tesis de maestro). Universidad Nacional de Cajamarca, Escuela de Posgrado. Cajamarca, Perú. Recuperado de: http://repositorio.unc.edu.pe/bitstream/handle/UNC/2170/Los\%20h\%c3\%a1 bitos $\% 20$ de $\% 20$ estudio $\% 20 \mathrm{y} \% 20$ su $\% 20$ relaci\%c3\%b3n\%20con\%20el\%20r endimiento $\% 20 \mathrm{acad} \% \mathrm{c} 3 \% \mathrm{a} 9$ mico $\% 20 \mathrm{de} \% 2010 \mathrm{~s} \% 20$ estudiantes $\% 20 \mathrm{de} \% 201$ a\%20Instit.pdf? sequence $=1 \&$ isAllowed $=y$

Mendoza Herrera, F. M. (2017). Relación entre los hábitos de estudio y el rendimiento académico en el área de comunicación de los estudiantes del nivel primario de la IE $N^{\circ} 14795$ Divino Jesús Misericordioso del Distrito de Bellavista. (Tesis de bachiller). Universidad Nacional Pedro Ruiz Gallo, Facultad de Ciencias Histórico Sociales y Educación. Sullana, Perú. Recuperado de: http://repositorio.unprg.edu.pe/bitstream/handle/UNPRG/1664/BC-TES-

TMP-517.pdf?sequence $=1 \&$ isAllowed $=y$

Muñoz-Muñoz, Y. E., and Troya-Baquerizo, M. P. (2017). Sistematización de Experiencias Prácticas de Investigación: Plan de talleres sobre las Técnicas de Estudio como una de las bases para el Aprendizaje Significativo en niños y niñas de $6^{\circ}$ Grado de Educación General Básica. Universidad de Guayaquil, Facultad de Ciencias Psicológicas. Guayaquil, Ecuador. Recuperado de: http://repositorio.ug.edu.ec/bitstream/redug/25817/1/Sistematizaci\%C3\%B3n $\% 20$ Ingrid\%20Mu\%C3\%B1oz\%20y\%20M\%C3\%A9lida\%20Troya\%20-.pdf

Pedreros Herrera, F. V. (2014). Clima social familiar de escolares con hábitos de 
estudio negativo de la I.E. Cesar Tello Rojas. Chimbote, 2014. (Tesis para el título profesional). Universidad Católica Los Ángeles. Facultad de Ciencias de la Salud. Chimbote, Perú.

Quintanilla Padilla, V. G., and Tacuri Carrazco, E. P. (2011). La inteligencia emocional como mejora en el rendimiento académico de los niños y niñas de cuarto año de educación básica del Colegio Militar $N^{o} 6$ "Combatientes de Tapi" del cantón Riobamba provincia de Chimborazo en el periodo lectivo 2010-2011. Universidad Estata de Bolívar, Facultad de Ciencias de la Educación, Sociales, Filosóficas y Humanísticas.

Quispe Mayta, U. (2018). Hábitos de lectura de las niñas y niños del ler grado en la I.E. $N^{\circ} 50322$ de Manco Inca Pucyura, Quillabamba 2017. (Tesis de bachiller). Universidad Nacional del Altiplano, Facultad de Ciencias de la Educación. Puno, Perú.

Reynoso Cantú, E. L. (2011). Factores que determinan el rendimiento escolar en el nivel secundario en el estado de Nuevo León. (Tesis doctoral). Universidad Autónoma de Nuevo León, Instituto de Investigaciones Sociales.

Rosales Fernandez, J. G. (2016). Estrés académico y hábitos de estudio en universitarios de la carrera de psicología de un centro de formación superior privada de Lima-sur. (Tesis de licenciatura). Universidad Autónoma del Perú, Facultad de Humanidades. Lima, Perú.

Sánchez-Balcázar, R. A., Flores-Nicolalde, B. C., and Flores-Nicolalde, F. (2016). Influencia de los hábitos de estudio en el rendimiento académico de los estudiantes de una institución de educación media ecuatoriana. Lat. Am. Phys. Educ., 10(1), 1-8.

Sánchez Balcázar, R. A., Flores Nicolalde, B. C., and Flores Nicolalde, F. (2016). Influencia de los hábitos de estudio en el rendimiento académico de los estudiantes de una institución de educación media ecuatoriana. Lat. Am. J. Phys. Educ, 10(1-7).

Sigchos Verdesoto, W. G. (2018). Los hábitos de estudio y el rendimiento académico en los estudiantes de quinto, sexto y séptimo de educación general básica en la Unidad Educativa "Avelina Lasso de Plaza", periodo 2016-2017. Universidad Central del Ecuador, Facultad de Filosofía Letras y Ciencias de la Educación. Quito, Ecuador.

Torres Bautista, J. M. (2017). Programa de hábitos y técnicas de estudio desde el aprendizaje significativo para I.E. Juan Luis Londoño de la cuesta: proyecto de pasantía. Universidad de Cundinamarca, Facultad de Ciencias Sociales y Humanas y Ciencias Políticas. Facatativá.

Vargas Hernández, M. M., and Montero Rojas, E. (2016). Factores que determinan el rendimiento académico en Matemáticas en el contexto de una universidad tecnológica : aplicación de un modelo de ecuaciones estructurales. Universitas Psychologica, 15(4), 1-12.

Vergel-Ortega, M., Martínez-Lozano, J. J., and Zafra-Tristancho, S. L. (2016). Factores asociados al rendimiento académico en adultos. Revista Científica, (25), 206-215. https://doi.org/10.14483/udistrital.jour.RC.2016.25.a4

Vilca Apaza, H. M., and Mamani Apaza, W. W. (2017). Los siete hábitos de los niños 
con alto rendimiento académico en Puno: análisis desde el contexto y tipo de gestión.COMUNI@CCIÓN, 8(1), 39-60. 